\title{
Coagulation status detection in breast cancer patients by using thrombelastography
}

\author{
Baolan Hao ${ }^{1}$, Hengwei Zhang ${ }^{2 *}$, Yuping Ye ${ }^{1}$, Xinjian Hao ${ }^{1}$, Yan Wang ${ }^{1}$ \\ ${ }^{1}$ Blood Transfusion Department; \\ ${ }^{2}$ Department of Breast Diseases, Affiliated Tumor Hospital of Zhengzhou University, Zhengzhou 450008, Henan, China.
}

\begin{abstract}
Many cancer patients are known to present in a hypercoagulable state, meaning an increased risk of thrombosis. To investigate hypercoagulable state in breast cancer (BC) patients, their coagulation status was compared with a benign disease group (control). The BC patients were divided into earlier stage (stage I and stage II ) and later stage (stage III and stage IV ). Thrombelastography (TEG) and other traditional coagulation tests were performed. The results showed that prothrombin time (PT) was significantly shortened and the levels of D-dimer, fibrinogen (Fib) and platelets (PLT) were significantly increased in the traditional BC group test $(P<0.05)$. According to TEG detection, the average level of blood clot formation time $(\mathrm{K})$ was significantly lower, while the Angle, MA and CI were significantly higher in the $\mathrm{BC}$ group than those in benign disease group $(P<0.05)$. There were 5 cases of lower extremity venous thrombosis in the breast cancer patients, coinciding with hypercoagulable state. The results showed that the BC patients had an increased hypercoagulable state, with hypercoagulability becoming more obvious in advanced stages. This study suggests that BC patients have an increased tendency for clot formation, and TEG monitoring could be a useful tool to predict the risk of thrombosis for clinical prevention and treatment.
\end{abstract}

Keywords: breast cancer, thrombelastography, coagulation, hypercoagulability, venous thromboembolism

\section{INTRODUCTION}

Abnormal coagulation function is always present in patients with malignant tumors ${ }^{[1]}$. Many studies have shown that cancer-related hypercoagulability can promote tumor development and metastasis in many cancers. The relationship between breast cancer $(\mathrm{BC})$ and the hemostatic system is increasingly recognized as an important regulator of the tumor's progression. Elements of the hemostatic system, including platelets, coagulation, and fibrinolysis, can

*Correspondence to: Hengwei Zhang, Department of Breast Diseases, Affiliated Tumor Hospital of Zhengzhou University, Zhengzhou 450008, China. E-mail: 13503814765@163.com

Conflict of interests: The authors declared no conflict of interests. mediate $\mathrm{BC}$ progression and metastasis, directly affecting the survival and prognosis of patients ${ }^{[2]}$. Thrombelastography(TEG) records the continuous profiles of whole blood coagulation by the measurement of viscoelastic changes associated with fibrin polymerization, and thereby provides a comprehensive assessment of haemostatic function, which is different from conventional coagulation tests ${ }^{[3]}$. In this study, we investigated 160 cases of BC using TEG detection, further verified by conventional coagulation tests; with 40 cases benign diseases as controls, in order to detect changes in coagulation (in patients with BC). Further knowledge in this area, we hope to improve the safe handling of risks associated with increased hypercoagulable states and thromboembolic complications. 


\section{MATERIALS AND METHODS}

\section{Instruments and equipment}

A Thrombelastograph Hemostasis Analyzer (TEG Model 5000) was provided by Haemonetics (MA,USA), while the IL ACL 9000 blood coagulation analyzer was provided by Instrumentation Laboratory (MA,USA).

\section{Tested patients}

200 female patients were enrolled from the Henan Cancer Hospital in China from May 1st, 2013 to September 30, 2016. Experimental group: 160 BC cases were diagnosed by pathology, age range: 25 to 83 years old, with an average age of 58 years. Control group: 40 cases of benign disease: 28 to 73 years old, with an average age of 55 years. Benign lesions included 28 cases of hyperplasia and hyperplasia with fibroadenoma, 8 cases of fibroadenoma, 4 cases of intraductal papilloma, were diagnosed by pathology. Two groups of patients were excluded due to the following factors: 1)pregnant, 2)on their menstrual period, 3)having a history of hemorrhage or thrombosis, 4)taking coagulation drugs and 5)suffering from other diseases within 3 months. These were all verified by blood sample before testing.

BC patients were briefly divided into 2 groups according to traditional TNM stage: Group A consisted of 59 cases, (average age: 64 years) clinical I and II stages. Group B had 101 cases, (average age: 57 years) stage III and IV . According to the AJCC cancer staging method, stage I includes: TINOM0, T0NlmiM0, T1NlmiM0; stage II includes: T0N1M0, T1N1M0, T2N0M0, T2N1M0, T3N0M0; stage III includes: T0N2M0, T1N2M0, T2N2M0, T3N1M0, T3N2M0, T4N0M0, T4N1M0, T4N2M0, AnyTN3M0; stage IV includes: AnyTanyNMl(T1 including Tlmi) ${ }^{[4]}$.

\section{Sample collection}

Blood samples were collected from the patients in the morning (after fasting). The detection of prothrombin time (PT), activated partial thromboplastin time (APTT), thrombin time (TT), fibrinogen (Fib), D-dimer, platelet (PLT) were measured within 2 hours and other blood samples were taken for TEG detection.

\section{Operating procedures of TEG-CK method}

All operations were conducted in strict accordance with the operating procedures. PT, APTT, TT were conducted by coagulation method; D-dimer by latex particle immune turbidimetric analysis; Fib by Clauss method.

TEG-CK method: Venous blood is mixed with sodium citrate (which acts as an anticoagulant), then $1 \mathrm{~mL}$ is drawn into a kaolin reagent cup, then slowly reversed up and down 5 times and placed at room temperature for $5 \mathrm{~min}$. The test cup is placed into the testing instrument, adding $20 \mu \mathrm{L}$ of calcium chloride, then $340 \mu \mathrm{L}$ citrated kaolin is slowly added. The appropriate program is then selected, with a running time generally of not less than $20 \mathrm{~min}$.

\section{The parameters adopted in the study}

Clotting time ( $\mathrm{R})$ : This is the time required for blood clots to begin forming, a process mainly influenced by coagulation factors; Blood clot formation time (K): Time spent for the blood clot to reach a certain level of strength; $\alpha$ (Angle): The speed of blood clot to reach this strength. The level of $\mathrm{K}$ and angle mainly reflect the level of fibrinogen; Maximum amplitude (MA): reflects the maximum strength or hardness of blood clots, mainly influenced by platelet function and quantity. CI value (CI) is the overall coagulation index calculated by the above indicators. A decreased $\mathrm{R}, \mathrm{K}$ and an increased Angle, MA and CI support hypercoagulability, otherwise hypocoagulability is recorded(Fig.1).

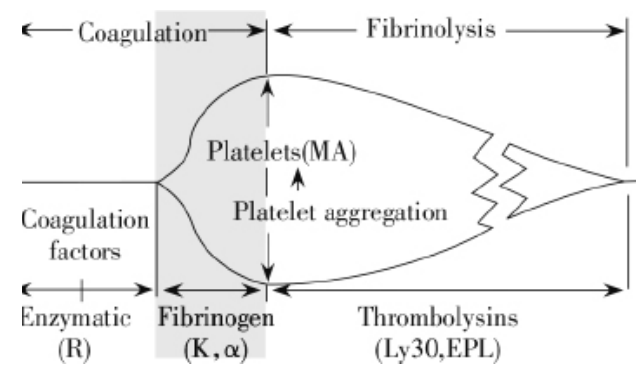

Fig.1 Schematic diagram of TEG

The normal TEG reference ranges are provided by the manufacturers. However, considering different characteristics brought about by place of origin and race, research data and conclusions based on the Chinese population were adopted. Because there are significant differences between male and female, the reference range of different gender groups was taken in combining various attributes which using the same instrument to detect ${ }^{[5-6]}$.

\section{Statistical analysis}

The data were measured in mean $\pm \mathrm{SD}$ (standard deviation) for statistical analysis. Data accorded with normal distribution were compared by $t$-test, or by rank sum test (Mann-Whitney U test); Numeration 
data were statistically analyzed by $\chi^{2}$ test, SPSS 21.0 statistical software was used and the significance was set at $P<0.05$.

\section{RESULTS}

Comparison of main parameters in TEG between BC groups and benign lesion group

A significantly reduced $\mathrm{K}$ value, increased Angle, MA and CI values indicated a hypercoagulable state in BC group in Table 1. The traditional coagulation test showed a shortened PT, and a heightened Ddimer, Fib and PLT in BC group $(P<0.05)$. Representative TEG traces of hypercoagulability in breast cancer patients and the hypercoagulable state in benign diseases patients are showed in Fig. 2.

\section{Comparison among groups at different stages}

$\mathrm{K}$ was significantly decreased, while Angle was significantly increased in B group $(P<0.05)$. MA and $\mathrm{CI}$ achieved a similarly significant difference ( $P=0.053$ and $P=0.058$ ) which still showed an increased tendency in group B for a hypercoagulable

Table 1 Measurement data of main parameters in two groups

\begin{tabular}{lccc}
\hline \multicolumn{1}{c}{ Parameters } & $\begin{array}{c}\text { Control group } \\
(n=40)\end{array}$ & $\begin{array}{c}\text { Experimental group } \\
(n=160)\end{array}$ & $P$ value \\
\hline R(min) & $3.58 \pm 1.07$ & $3.42 \pm 0.78$ & $>0.05$ \\
K(min) & $1.45 \pm 0.33$ & $1.31 \pm 0.35$ & 0.007 \\
Angle(deg) & $69.67 \pm 4.89$ & $71.25 \pm 6.48$ & 0.021 \\
MA(mm) & $63.24 \pm 3.77$ & $65.71 \pm 3.81$ & 0.034 \\
CI & $2.35 \pm 1.17$ & $2.95 \pm 1.05$ & 0.002 \\
PT(s) & $12.22 \pm 2.89$ & $11.19 \pm 3.26$ & 0.046 \\
APTT(s) & $33.11 \pm 3.94$ & $32.89 \pm 6.72$ & $>0.05$ \\
Fib $(\mathrm{g} / \mathrm{L})$ & $2.83 \pm 1.48$ & $3.41 \pm 2.03$ & 0.027 \\
TT(s) & $15.97 \pm 3.79$ & $15.28 \pm 4.03$ & $>0.05$ \\
D-D $(\mathrm{ng} / \mathrm{mL})$ & $262.88 \pm 63.51$ & $301.37 \pm 82.79$ & 0.003 \\
PLT $\left(10^{9} / \mathrm{L}\right)$ & $129.76 \pm 39.57$ & $154.28 \pm 65.26$ & 0.001 \\
\hline
\end{tabular}

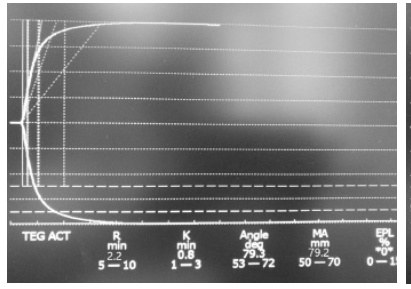

A

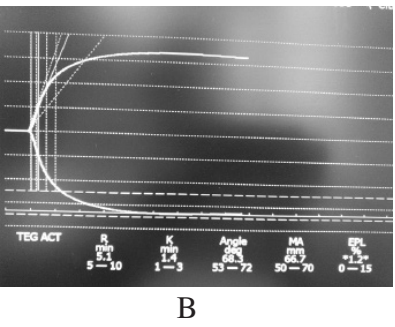

B
Fig.2 Representative TEG traces of hypercoagulability in a breast cancer patient and a patient with benign disease.A: Representative TEG traces of hypercoagulability in a breast cancer patient: B: Representative TEG traces of the hypercoagulable state in a patient with benign disease

state in Table 2. This suggested that an increased hypercoagulable state could be more obvious at later stages of BC.

\section{Comparison of the number of individual measurements which were beyond the nor- mal range in two groups}

In the distribution of abnormal cases showed in Table 3, there were significant differences in $\mathrm{K}, \mathrm{An}-$ gle and CI values from two groups $(P<0.05)$. In the indexes of $\mathrm{K}$, Angle and $\mathrm{CI}$, the rates of high coagulation / total number of cases in the experimental group were $34 / 160(21 \%), 48 / 160(30 \%)$ and 77/160 $(48 \%)$ respectively, as well as a significant increase over the control group which reached $3 / 40(7.5 \%)$, $6 / 40(15 \%)$ and $11 / 40(27.5 \%)$ respectively.

There were 5 cases of lower extremity venous thrombosis in breast cancer patients showing a hypercoagulable state (Taking CI value as the standard), which were relieved after the thrombolysis and anticoagulation treatment. No thrombosis occurred in the benign breast lesion group. The hypercoagulability was thought to increase the risk of thrombosis in breast cancer patients.

Table 2 Measurement data of main parameters in TEG in group A and B

\begin{tabular}{lcccccc}
\hline Clinical stages & $n$ & $\mathrm{R}(\mathrm{min})$ & $\mathrm{K}(\mathrm{min})$ & Angle $(\mathrm{deg})$ & $\mathrm{MA}(\mathrm{mm})$ & CI \\
\hline Group A & 101 & $3.44 \pm 0.78$ & $1.34 \pm 0.41$ & $71.44 \pm 4.37$ & $65.11 \pm 4.11$ & $2.84 \pm 1.10$ \\
Group B & 59 & $3.39 \pm 0.83$ & $1.20 \pm 0.31$ & $72.80 \pm 3.72$ & $66.52 \pm 4.01$ & $3.12 \pm 1.27$ \\
$P$ value & & 0.765 & 0.013 & 0.043 & 0.053 & 0.058 \\
\hline
\end{tabular}

Table 3 Distribution of abnormal cases in benign and malignant groups

\begin{tabular}{|c|c|c|c|c|c|c|c|c|c|c|c|c|c|c|c|}
\hline \multirow{3}{*}{ Parameters } & \multicolumn{3}{|c|}{$\mathrm{R}(2.8 \sim 7.2 \mathrm{~min})$} & \multicolumn{3}{|c|}{$\mathrm{K}(1.1 \sim 2.6 \mathrm{~min})$} & \multicolumn{3}{|c|}{ Angle(55.8 73.8 deg) } & \multicolumn{3}{|c|}{$\operatorname{MA}(54.1 \sim 67.9 \mathrm{~mm})$} & \multicolumn{3}{|c|}{$\mathrm{CI}(-1.8 \sim 3.0)$} \\
\hline & \multirow{2}{*}{$N$} & \multicolumn{2}{|c|}{$\mathrm{A}$} & \multirow{2}{*}{$N$} & \multicolumn{2}{|c|}{ A } & \multirow{2}{*}{$N$} & \multicolumn{2}{|c|}{$\mathrm{A}$} & \multirow{2}{*}{$N$} & \multicolumn{2}{|c|}{ A } & \multirow{2}{*}{$N$} & \multicolumn{2}{|c|}{$\mathrm{A}$} \\
\hline & & I & II & & $\mathrm{I}$ & II & & $\mathrm{I}$ & II & & I & II & & I & II \\
\hline Benign & 32 & 8 & 0 & 37 & 3 & 0 & 33 & 6 & 1 & 35 & 5 & 0 & 29 & 11 & 0 \\
\hline Malignant & 131 & 29 & 0 & 126 & 34 & 0 & 112 & 48 & 0 & 150 & 49 & 1 & 83 & 77 & 0 \\
\hline$\chi^{2}$ value & \multicolumn{3}{|c|}{0.075} & \multicolumn{3}{|c|}{4.013} & \multicolumn{3}{|c|}{7.356} & \multicolumn{3}{|c|}{4.510} & \multicolumn{3}{|c|}{5.530} \\
\hline$P$ value & \multicolumn{3}{|c|}{0.785} & \multicolumn{3}{|c|}{0.045} & \multicolumn{3}{|c|}{0.025} & \multicolumn{3}{|c|}{0.105} & \multicolumn{3}{|c|}{0.019} \\
\hline
\end{tabular}

$\mathrm{N}$ : Normal, the cases whose measurements were in the normal range;A: Abnormal, the cases whose measurements were not in the normal range;

I : Abnormal cases supporting hypercoagulability; II : Abnormal cases supporting hypocoagulability. 


\section{DISCUSSION}

A number of clinical and laboratory studies have showed that most patients with malignant tumors are associated with abnormal coagulation function ${ }^{[1]}$. The coagulation and anticoagulation system reside in a state of dynamic balance in normal physiological status, while malignant tumor cells can affect the hemorheology and blood microenvironment to change this balance. Elevated coagulation activity or reduced anticoagulation activity results in hypercoagulability ${ }^{[7]}$. At the same time, the hypercoagulable state of the body can promote tumor growth, invasion and metastasis ${ }^{[8]}$. Previous research on the coagulation function of cancer is mainly based on traditional coagulation experiments such as PT, APTT, TT, blood routine and D-dimer, while are still not quite as advanced as TEG. TEG assess the coagulation process and provides a global coagulation index for whole blood so that more physiologically relevant than assays of isolated haemostatic components ${ }^{[9]}$. Many previous studies using TEG have shown that blood hypercoagulability was confirmed in $\mathrm{BC}^{[9]}$, colon cancer ${ }^{[9]}$ and lung cancer ${ }^{[10]}$.

In this study, the TEG results showed significant difference in K, Angle, MA and CI, but no significant difference in R. The decreased K, increased Angle, MA and CI, all supported the hypercoagulable state of $\mathrm{BC}$ compared with benign diseases. The comparison between A group and B group proved that hypercoagulability is more obvious in advanced stages in breast cancer. The parameter of $\mathrm{R}$ is mainly affected by the plasma coagulation factors, while the $\mathrm{K}$ and Angle mainly reflects the level of Fib. MA is mainly influenced by the function and quantity of platelet and the CI value is the global coagulation index, reflecting the overall coagulation condition. Such a result showed that common hypercoagulability in $\mathrm{BC}$ is probably due to the increased platelet and fibrinogen level(decreased K, increased Angle and MA value), instead of the increased plasma coagulation factors( $\mathrm{R}$ level is normal). Also, the elevated level of platelet and Fib seems to still play a leading role in aggravated hypercoagulability, coinciding with the development of the disease. In Inder Lal's review $^{[2]}$ and Krenn-Pilko Sabine's article ${ }^{[11]}$, an increased level of platelet and Fib was showed to play very important roles in coagulation activation. While an elevated preoperative Fib level may represent an independent prognostic marker for survival in $\mathrm{BC}$ patients. These all appeared to be further confirmed in our study, which found more positive items such as $\mathrm{K}$ and $\mathrm{CI}$ compared to the previous studies' results, which means there is more evidence to support the idea of hypercoagulability, however our $\mathrm{R}$ value remained unaltered. Such results are also verified by the results of the traditional coagulation tests which showed increased PLT, Fib and D-dimer. The rate of abnormal results showed the hypercoagulability in $48 \%$ of cases(77/160) in terms of CI value(the overall coagulation index) which was much higher than the 2/21(9\%) reported by Francis JL using the same detection method (TEG) ${ }^{[5]}$.

A significant body of evidence has established that coagulation activation is not just an epiphenomenon in $\mathrm{BC}$ progression, but also an important regulator of malignant transformation, tumor angiogenesis and metastasis $^{[2]}$. The combination of an increase in RBC aggregation and plasma viscosity impairs bloodflow-properties and may induce hypoxia in the micr ocirculation that favors thrombosis, the settlement of tumor-cells and thus metastasis ${ }^{[12]}$. The hypercoagulable state in cancer involves several complex interdependent mechanisms, including interaction among cancer cells, host cells, and the coagulation system ${ }^{[1]}$. For tumor cells, some procoagulant phenotypes are able to stimulate coagulation through the activation of oncogenes ${ }^{[13]}$. For the host cell, the endothelial inflammatory response leads platelets and leukocytes to signal tumor cells to release procoagulant factors, of which the most important procoagulant protein is tissue factor(TF $)^{[14]}$. With the releasing of procoagulant factor, these lead to coagulation activation.

This pathological mechanism is complex and still need to be researched furtherly. The consequences of hypercoagulability often result in venous thromboembolism (VTE), which can cause severe complications ${ }^{[8]}$. Cancer patients have a 4 fold increased risk of VTE compared with the general population, and this is associated with significant morbidity and mortality ${ }^{[2,15-16]}$. Due to the decrease in activity, vascular catheterization and postoperative inflammatory response, patients with hypercoagulability are prone to venal thrombosis of the upper and lower extremities, embolism of the catheter, or pulmonary embolism, any of which can be fatal. In addition, chemotherapy or surgical operation may also enhance hypercoagulability and initiate $\mathrm{VTE}^{[17-18]}$. In recent years, our hospital has also reported the death of cancer patient caused by thromboembolism. Although more data still need to be further analyzed, this test also confirmed that the detection of TEG can help predict the risk of thrombosis in patients. TEG is applicable for patients to provide important monitoring ${ }^{[18-19]}$. Clinicians are well advised to use anticoagulation therapy for patients at risk for high coagulation, which can 
effectively prevent VTE and improve the safety of perioperative period or other treatment ${ }^{[14,20]}$. But beyond that, improvements in blood fluidity and thus oxygen transfer in tumor-vascular-microcirculation may increase the susceptibility of systemic anticancer therapy through anticoagulation therapy ${ }^{[12]}$.

\section{CONCLUSION}

Breast cancer patients often show an increased hypercoagulable state when compared with benign diseases patients, which often becomes more obvious in advanced stages. Elevated levels of platelet and fibrinogen played a more major role in hypercoagulability when compared with plasma coagulation factors. TEG monitoring could be a useful tool to predict the risk of thrombosis for patients with hypercoagulability for clinical prevention and treatment.

\section{Acknowledgements}

The authors recognized and thank all the participants of the blood transfusion department in our hospital who had successfully conducted the study. We sincerely thank Dr. Jian Zhong and Dr. Yingjie Han for reviewing this manuscript.

\section{References}

[1] Yamashita Y. Hypercoagulable state in cancer[J]. Rinsho Byori,2015,63(12):1435-1442

[2] Lal I, Dittus K, Holmes CE. Platelets, coagulation and fibrinolysis in breast cancer progression[J]. Breast Cancer Res,2013,15(4):207

[3] Reikvam H, Steien E, Hauge B, et al. Thrombelastography [J]. Transfus Apher Sci,2009,40(2):119-123

[4] Edge SB,Byrd DR,Compton CC,et al. AJCC cancer staging manual [M]. $7^{\text {th }}$ ed. New York: Springer,2010,347-376.

[5] Wang Y, Dou M, Lin Y, et al. To construct normal reference range for thromboelastography in Fuzhou region among healthy adults[J]. Fujian Med $J$ (in Chinese),2012,34(6):58-6

[6] Yi SH, Lin J, Luo QJ, et al. Normal reference range for thromboelastography in Lanzhou region healthy adult volunteers of Gansu Province[J]. Med J West China,2016,28(2):266-268

[7] Gong WJ. Mechanism and prophylaxis of cancerassociated venous thromboembolism[J]. J Inter Oncol, 2010,37(4):284-286
[8] Anderson JA, Weitz JI. Hypercoagulable states[J]. Clin Chest Med, 2010,31(4):659-673

[9] Francis JL, Francis DA, Gj G. Assessment of hypercoagulability in patients with cancer using the Sonoclot Analyzer and thromboelastography[J]. Thromb Res, 1994,74(4):335-346

[10] Davies NA, Harrison NK, Sabra A, et al. Application of ROTEM to assess hypercoagulability in patients with lung cancer[J]. Thromb Res,2015,135(6):1075-1080

[11] Krenn-Pilko S, Langsenlehner U, Stojakovic T, et al. An elevated preoperative plasma fibrinogen level is associated with poor disease-specific and overall survival in breast cancer patients[J]. Breast,2015,24(5):667-672

[12] von Tempelhoff GF, Nieman F, Heilmann L, et al. Association between blood rheology, thrombosis and cancer survival in patients with gynecologic malignancy[J]. Clin Hemorheol Microcirc, 2000,22(2):107-130.

[13] Falanga A, Schieppati F, Russo D. Cancer tissue procoagulant mechanisms and the hypercoagulable state of patients with cancer[J]. Semin Thromb Hemost,2015,41(7):756-764

[14] Ay C, Pabinger I, Cohen AT. Cancer-associated venous thromboembolism:Burden, mechanisms, and management[J]. Thromb Haemost, 2017,117(2):219230

[15] Hisada Y, Geddings JE, Ay C, et,al. Venous thrombosis and cancer: from mouse models to clinical trials $[\mathrm{J}]$. $J$ Thromb Haemost,2015,13(8):1372-1382.

[16] Medioni J, Guastalla JP, Drouet L. Thrombosis and breast cancer: incidence, risk factors, physiopathology and treatment[J]. Bull Cancer,2012,99(2):199-210

[17] Kirwan CC, McCollum CN, McDowell G, et al. Investigation of proposed mechanisms of chemotherapyinduced venous thromboembolism: endothelial cell activation and procoagulant release due to apoptosis[J]. Clin Appl Thromb Hemost,2015,21(5):420-427.

[18] Allen CJ, Karcutskie CA, Zebib L, et al. Coagulation changes following combined ablative and reconstructive breast surgery[J]. Plast Reconstr Surg,2016,137(6):923E930E

[19] De Pietri L, Montalti R, Begliomini B, et al. Thromboel astographic changes in liver and pancreatic cancer surgery: hypercoagulability, hypocoagulability or normocoagulability?[J]. Eur J Anaesthesiol,2010,27(7):608-616

[20] Hollenhorst MA, Battinelli ET, Hypercoagulable States. And anticoagulants[J]. Prim Care,2016,43(4):619-635

(Received 10 October 2018, Revised 21 November 2018, Accepted 02 December 2018) 


\section{Antibody}

安迪溥拮

\section{JiangSu LIBO Medicine Biotechnology Co.,Ltd.}

\section{Diagnosis of Hemolytic Disease of the Newborn}

\section{ABO、Rh(D) Antigen Testing Card Newborn}
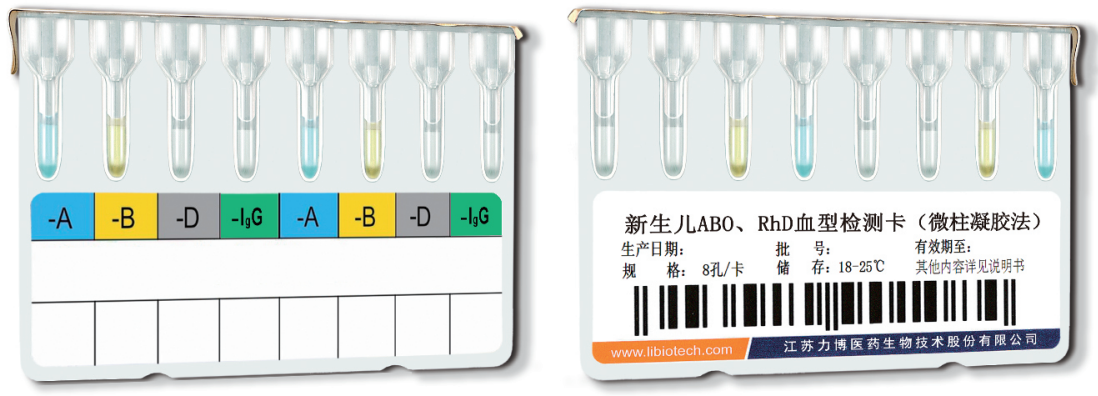

Intended Use :

The product is suitable for clinical tests on $\mathrm{ABO}$ and $\mathrm{Rh}(\mathrm{D})$ blood typing and direct antiglobulin test (DAT) for newborns and suspected hemolytic disease patients. It is only applicable for clinical test, but not for blood screening.

\section{Case :}
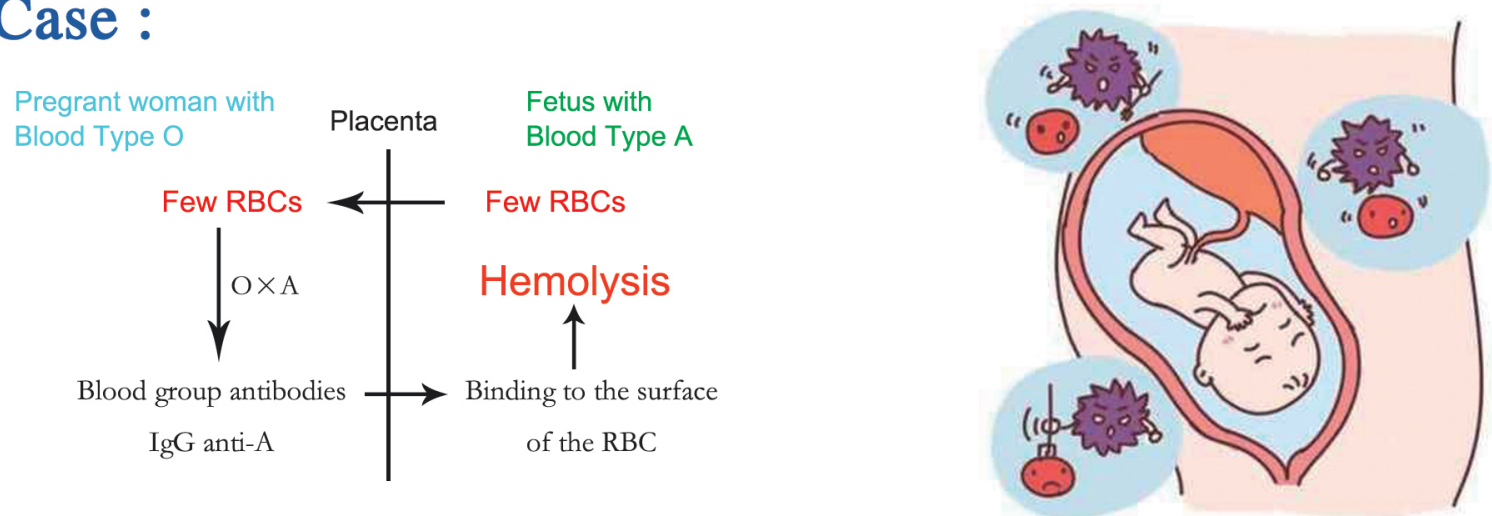

\section{Antibodl 安画溥君 \\ JiangSu LIBO Medicine Biotechnology Co.,Ltd.}

Address: No 78 West Dongsheng Road, Jiangyin, Jiangsu214400, P.R. China

Web Site: www.libiotech.com

Sales Tel: +86-510-86990618、+86-510-86990633

Tech Tel: +86-510-86990608、+86-510-86990655

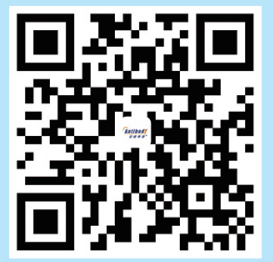

